\title{
REST, regulated by RA through miR-29a and the proteasome pathway, plays a crucial role in RPC proliferation and differentiation
}

\author{
Yuyao Wang ${ }^{1,2}$, Dandan Zhang ${ }^{1,2}$, Zhimin Tang ${ }^{1,2}$, Yi Zhang ${ }^{1,2}$, Huiqin Gao ${ }^{1,2}, \mathrm{Ni} \mathrm{Ni}^{1,2}$, Bingqiao Shen ${ }^{1,2}$, Hao Sun ${ }^{1,2}$ and \\ Ping $\mathrm{Gu}^{1,2}$
}

\begin{abstract}
One of the primary obstacles in the application of retinal progenitor cells (RPCs) to the treatment of retinal degenerative diseases, such as age-related macular degeneration (AMD) and retinitis pigmentosa (RP), is their limited ability to proliferate and differentiate into specific retinal neurons. In this study, we revealed that repressor element-1silencing transcription factor (REST), whose expression could be transcriptionally and post-transcriptionally mediated by retinoic acid (RA, one isomeride of a vitamin A derivative used as a differentiation-inducing agent in many disease treatments), plays a pivotal role in the regulation of proliferation and differentiation of RPCs. Our results show that direct knockdown of endogenous REST reduced RPC proliferation but accelerated RPC differentiation toward retinal neurons, which phenocopied the observed effects of RA on RPCs. Further studies disclosed that the expression level of REST could be downregulated by RA not only through upregulating microRNA (miR)-29a, which directly interacted with the $3^{\prime}$-untranslated region (3'-UTR) of the REST mRNA, but also through promoting REST proteasomal degradation. These results show us a novel functional protein, REST, which regulates RPC proliferation and differentiation, can be mediated by RA. Understanding the mechanisms of REST and RA in RPC fate determination enlightens a promising future for the application of REST and RA in the treatment of retinal degeneration diseases.
\end{abstract}

\section{Introduction}

Retinal progenitor cells (RPCs) are a side branch of neural progenitor cells that sustain the undifferentiated status with the potential for self-renewal and differentiation into retinal neuronal cells and have great potential to treat retinal degenerative diseases, such as age-related macular degeneration (AMD) and retinitis pigmentosa $(\mathrm{RP})^{1-3}$. Although RPCs are identified as one source of

\footnotetext{
Correspondence: Bingqiao Shen (shenbingqiao.123@163.com) or

Hao Sun (sunha06666@126.com) or Ping Gu (guping2009@126.com)

${ }^{1}$ Department of Ophthalmology, Ninth People's Hospital, Shanghai Jiao Tong

University School of Medicine, 200011 Shanghai, P.R. China

${ }^{2}$ Shanghai Key Laboratory of Orbital Diseases and Ocular Oncology, 200011

Shanghai, P.R. China

These authors contributed equally: Yuyao Wang, Dandan Zhang, Zhimin Tang.

Edited by B. Joseph
}

replaceable multipotent progenitor cells that can be derived from both embryonic and adult mammalian retina, the limited proliferation and differentiation capacity toward specific retinal neurons in vitro restricts their future clinical applications ${ }^{4-6}$. This emphasizes the importance of a better understanding of the mechanisms controlling RPC proliferation and differentiation.

Repressor element-1 (RE-1) is known as a piece of conserved DNA sequence locating in the transcriptional regulatory regions of many neuronal genes ${ }^{7-9}$. Repressor element-1-silencing transcription factor (REST, also known as neuron-restrictive silencer factor, NRSF) is a zinc-finger protein, which interacts with RE-1, exerting a gene silencing effect ${ }^{10-13}$. Generally, during embryonic

\section{(c) The Author(s) 2018}

(c) (i) Open Access This article is licensed under a Creative Commons Attribution 4.0 International License, which permits use, sharing, adaptation, distribution and reproduction c. in any medium or format, as long as you give appropriate credit to the original author(s) and the source, provide a link to the Creative Commons license, and indicate if changes were made. The images or other third party material in this article are included in the article's Creative Commons license, unless indicated otherwise in a credit line to the material. If material is not included in the article's Creative Commons license and your intended use is not permitted by statutory regulation or exceeds the permitted use, you will need to obtain permission directly from the copyright holder. To view a copy of this license, visit http://creativecommons.org/licenses/by/4.0/. 
stem cells (ESCs) differentiation into neural progenitor cells (NPCs), REST is downregulated by proteasomal degradation. When transitioning from progenitor to mature neurons, REST and its corepressors dissociate from neuronal gene chromatin accompanied by its transcriptional repression ${ }^{11,12}$. The gradually decreased expression of REST is necessary during neuron differentiation and REST overexpression leads to neuronal gene expression disorder and axon pathfinding mistakes ${ }^{14}$. The literature suggests that REST plays an important role in the generation of functional mature neurons. Recently, REST has become a hot topic in the field of the central nervous system (CNS), as its expression levels in neurons closely correlate with recognition, longevity and neuropathological processes ${ }^{15}$. REST expression accumulates in seizures and epilepsy ${ }^{16}$, ischemia ${ }^{17,18}$ and alcoholism ${ }^{19}$, and relative phenotypes of these diseases can be attenuated by blocking REST function. On the other hand, in aging and Alzheimer's disease, REST exerts a neuronal protective effect through spurring stress response genes expression and suppressing genes that facilitate cell death and disease pathology ${ }^{15}$. Although REST has been researched extensively in the CNS, its role in neural retina remains to be explored. As RPCs are one of the promising cell resources in the treatment of retinal degeneration diseases, it is worth detecting the role of REST in RPC fate determination.

Retinoic acid (RA) is a vitamin A derivative that is synthesized by the enzyme retinaldehyde dehydrogenase ${ }^{20}$ and plays a major role during the early development of the nervous system ${ }^{21}$. The mechanisms of RA that influence cell fates during the development of the nervous system have been investigated in many studies, including in the retina ${ }^{22-27}$. All-trans RA (ATRA) is an isomeride of RA and has been used to treat many kinds of diseases, that is, acne or other disorders of keratinization $^{28}$, and, most importantly, to treat cancer, as it generally inhibits tumor cells proliferation and induces their differentiation and apoptosis ${ }^{29}$. The most established use of ATRA is in the induction of remission in patients with acute promyelocytic leukemia (APL) ${ }^{30}$. Moreover, the use of ATRA-based chemotherapy in the induction phase of the treatment of APL was approved by the Food and Drug Administration (FDA), which made it possible to use ATRA as a potential medicine to treat retinal degeneration.

The microRNA (miRNA) is one of small non-coding RNAs, which downregulate gene expression mainly through binding to their $3^{\prime}$-untranslated region ( $3^{\prime}$-UTR), causing translation repression or mRNA degradation ${ }^{31}$. miR-29a is one member of miR-29 family, which are involved in normal tissue differentiation ${ }^{32}$, including skeletal myogenesis ${ }^{33}$, osteoblast differentiation ${ }^{34}$ and neuron differentiation ${ }^{35,36}$. In addition, our previous study demonstrated that miR-29a plays an important role during RPC proliferation and differentiation by targeting RBM8 ${ }^{37}$.

In this study, the role of REST in the proliferation and differentiation of RPCs was investigated. We further identified the endogenous REST expression levels that could be mediated by exogenous RA through promoting miR-29a expression (to inhibit REST mRNA levels) and accelerating REST proteasomal degradation.

These results provide us with a deeper understanding of the mechanisms regarding how REST regulate RPCs' selfrenewal and differentiation and the promising field of using RA in the clinical treatment of retinal degeneration diseases.

\section{Materials and methods \\ Cell isolation and culture}

RPCs were obtained from the fresh neural retina that isolated from postnatal day $1 \mathrm{C} 57 \mathrm{BL} / 6 \mathrm{mice}^{38}$, and cells from the fifth passage or sixth passage were used in this study. The proliferation medium contains $20 \mathrm{ng} / \mathrm{ml}$ recombinant epidermal growth factor (EGF, Invitrogen, Carlsbad, CA, USA), advanced Dulbecco's modified Eagle's medium (DMEM)/F12 (Invitrogen), $2 \mathrm{mM}$ L-glutamine (Invitrogen) and 1\% N2 neural supplement (Invitrogen) $^{6}$. The differentiation medium consists of $10 \%$ fetal bovine serum (FBS, Invitrogen), advanced DMEM/F12 (Invitrogen), 1\% N2 neural supplement and without EGF.

All of the animals were handled according to the animal usage standards of the Association for Research in Vision and Ophthalmology (ARVO) and following approval by the Animal Research Committee of the Ninth People's Hospital, Shanghai Jiao Tong University School of Medicine.

\section{Drug treatment}

In our pre-experiment, cells were incubated with RA (Sigma-Aldrich, St. Louis, MO, USA) with a concentration from 10 to $500 \mathrm{ng} / \mathrm{ml}$, and $100 \mathrm{ng} / \mathrm{ml}$ was chosen as the desired concentration (data not shown). RA was dissolved in dimethyl sulfoxide (DMSO, Sigma-Aldrich) with recommended storage at a concentration of $10 \mathrm{mM}$ and stored at $-20^{\circ} \mathrm{C}$ in the dark. Individual aliquots were diluted to the appropriate concentration in the culture medium.

RPCs were cultured with $100 \mathrm{ng} / \mathrm{ml} \mathrm{RA}$ in proliferation or differentiation medium according to the different experimental objectives. To detect the effects of RA on REST expression, RPCs were treated with RA $(100 \mathrm{ng} / \mathrm{ml})$ under proliferation medium for 3 days followed by the addition of $20 \mu \mathrm{M}$ MG-132 (Sigma-Aldrich) for another 8 h. RPCs treated with or without RA for 3 days were used as the control. 


\section{Luciferase assay}

For the construction of the luciferase reporter vector, the 3'-UTR-wt (Genechem, Shanghai, China) of the REST mRNA was composed of a 713-bp fragment of the REST mRNA 3'-UTR containing the predicted miR-29a binding site (positions 561-567), and the REST mRNA 3'-UTR$\mathrm{mu}$ (Genechem) was composed of REST mRNA 3'-UTR containing the predicted miR-29a mutant sequence.

HEK 293 cells were transfected with the miR-29a mimics or control miRNA. After $48 \mathrm{~h}$, cells were used for luciferase assays according to the manufacturer's instruction.

\section{Transfection}

Small interfering RNAs, including siREST and negative control, miR-29a oligonucleotides, including the miR-29a inhibitor, miR-29a mimics and the negative control, were synthesized by Biomics Biotech Co., Ltd (Nantong, China). Before transient transfections, RPCs were cultured under differentiation medium to allow for attachment for 8-12 h. Then, Lipofectamine 2000 (Invitrogen) and oligonucleotides (siREST, miR-29a inhibitor, and negative control) were blended with Opti-MEM (Invitrogen) medium for $5 \mathrm{~min}$, respectively. Following incubation of Lipofectamine 2000Opti-MEM with oligonucleotide-Opti-MEM for $20 \mathrm{~min}^{38}$. RPCs were incubated with this mixture in plates for $6-8 \mathrm{~h}$ before it was replaced by proliferation or differentiation medium. Oligonucleotides were repeatedly transfected into RPCs every 3 days under differentiation culture conditions. The oligonucleotide sequence of siREST was as follows: 5'- GCGCUAAGAAGUUCUUUGUdTdT-3'.

\section{Quantification of the cells' viability}

The different groups of RPCs were cultured in 96-well plates with the density of $1 \times 10^{4}$ per well. RPCs were incubated with Lipofectamine 2000 (control group) or siREST (siREST group); DMSO (control group) or $100 \mathrm{ng} /$ ml RA (RA group); and Lipofectamine 2000 plus DMSO (control group), $100 \mathrm{ng} / \mathrm{ml} \mathrm{RA}$ (RA group), $100 \mathrm{ng} / \mathrm{ml} \mathrm{RA}$ plus miR-29a inhibitor (RA + miR-29a inhibitor group) or miR-29a (miR-29a group) under proliferation medium, and the cell counting kit (CCK-8, Dojindo, Kumamoto, Japan) was used to quantitate the number of viable cells. The CCK-8 solution was added to each well at different time points: $0 \mathrm{~h}, 24 \mathrm{~h}, 48 \mathrm{~h}$ and $72 \mathrm{~h}$. The absorbance at $450 \mathrm{~nm}$ was measured using a microplate reader (ELX800, BioTek, Vermont, USA) after incubation for another $4 \mathrm{~h}$ at $37^{\circ} \mathrm{C}$.

\section{Reverse transcription and quantitative polymerase chain reaction (qPCR)}

In all, $1 \mu \mathrm{g}$ of total RNA was extracted and reverse transcribed according to previous study ${ }^{38}$. Then, the qPCR was performed with a 7500 Real-Time PCR Detection System (Applied Biosystems, Irvine, CA, USA) using $2 \mu \mathrm{l}$ of 10-fold diluted complementary DNA, $10 \mu \mathrm{l}$ of 2\%miRcute miRNA premix (Tiangen Biotech Co.) or $10 \mu \mathrm{l}$ of $2 \times$ Power SYBR Green PCR Master Mix (Applied Biosystems), $2 \mu \mathrm{l}(300 \mathrm{nM})$ primers of target genes (Table 1) and the rest was supplemented with nuclease-free water (Invitrogen) to make the reaction volume to be $20 \mu$ l. The relative mRNA was analyzed using the Pfaffl method after 40 cycles of amplification. The relative mRNA was expressed as the fold change relative to sonRNA-202 or $\beta$ actin, which was used as an endogenous normalization control for the miRNA and mRNA, respectively.

Table 1 Primers used for qPCR

\begin{tabular}{llllll}
\hline Gene & Accession no. & Forward $\left(\mathbf{5}^{\prime} \mathbf{-} \mathbf{3}^{\prime}\right)$ & Reverse $\left(\mathbf{5}^{\prime} \mathbf{-} \mathbf{3}^{\prime}\right)$ & Annealing temperature $\left.{ }^{\circ} \mathbf{C}\right]$ & Product size (base pairs) \\
\hline Ki-67 & X82786 & cagtactcggaatgcagcaa & cagtcttcaggggctctgtc & 60 & 170 \\
Nestin & NM_016701 & aactggcacctcaagatgt & tcaagggtattaggcaagggg & 60 & 235 \\
Pax-6 & NM_013627 & agtgaatgggcggagttatg & acttggacgggaactgacac & 60 & 269 \\
B3-Tublin & NM_023279 & cgagacctactgcatcgaca & cattgagctgaccagggaat & 60 & 152 \\
Rhodopsin & NM_145383 & tcaccaccaccctctacaca & tgatccaggtgaagaccaca & 60 & 216 \\
Recoverin & NM_009038 & atggggaatagcaagagcgg & gagtccgggaaaaacttggaata & 60 & 184 \\
Brn3a & NM_0111434 & cgctctcgcacaacaacatga & ttcttctcgccgccgttga & 60 & 121 \\
Calbindin & NM_009788 & ggcttcatttcgacgctgac & acgtgagccaactctacaattc & 60 & 184 \\
REST & NM_005612 & gtgcgaactcacacaggaga & aagaggtttaggcccgttgt & 60 & 201 \\
Casepase-3 & NM_004346 & catggaagcgaatcaatggact & ctgtaccagaccgagatgtca & 60 & 139 \\
B-Actin & NM_007393 & agccatgtacgtagccatcc & ctctcagctgtggtggtgaa & 60 & 152 \\
\hline
\end{tabular}




\section{Western blot analysis}

Western blot analyses were performed as previously described $^{38}$. Various antibodies, including rabbit monoclonal anti-REST (Abcam, Cambridge, UK), mouse antinestin (BD, San Jose, CA, USA), rabbit monoclonal antiPax-6 (Biolegend, San Diego, CA, USA), mouse monoclonal anti- $\beta 3$-tubulin (Millipore, Billerica, MA, USA), mouse monoclonal anti-rhodopsin, rabbit polyclonal antirecoverin (Millipore), mouse monoclonal anti-Brn3a (Millipore) and mouse monoclonal anti-Caspase-3 (Santa Cruz, California, USA) were diluted 1:1000, and mouse anti- $\beta$-actin (Sigma-Aldrich) was diluted 1:5000. The membranes were incubated with 1:5000 dilutions of DyLightTM680-conjugated goat anti-mouse or goat antirabbit secondary antibodies (Sigma-Aldrich), and analyzed by Odyssey V 3.0 image scanning (LI-COR, Lincoln, NE, USA).

\section{Immunocytochemistry}

Digested RPCs were seeded on glass coverslips (VWR, West Chester, PA, USA) in 24-well plates. Cells treated with siREST or RA were cultured for 3 or 7 days in proliferation or differentiation medium, respectively. Then, cells were fixed with $4 \%$ paraformaldehyde (SigmaAldrich) for $20 \mathrm{~min}$ and treated with blocking reagent consisting of $10 \%$ of goat serum (Sigma-Aldrich), $0.3 \%$ of Triton-100 (Sangon, Shanghai, China) in phosphatebuffered saline buffer. After $1 \mathrm{~h}$, cells were incubated with various antibodies, including rabbit polyclonal antiREST (Abcam), mouse monoclonal anti-Ki-67 (BD), mouse monoclonal anti-Nestin (Millipore), mouse monoclonal anti- $\beta 3$-tubulin (Millipore) and rabbit polyclonal anti-recoverin (Millipore) overnight at $4{ }^{\circ} \mathrm{C}$ and incubated with Alexa Fluor546-goat anti-mouse/rabbit secondary antibodies (BD) in the dark for $1 \mathrm{~h}$. Cell nuclei were counterstained with Hoechst (Invitrogen). Negative controls were performed in parallel but without primary antibodies. For 5-Bromo-2-deoxyuridine (BrdU) incorporation, the anti-BrdU antibody (Cell Signaling Technology, Danvers, MA, USA) was used to detect proliferating cells, which was treated with $10 \mathrm{mM}$ BrdU (Sigma-Aldrich) for $10 \mathrm{~h}$. Immunoreactive cells were visualized by the fluorescence microscope (Olympus BX51, Japan) and images of six random fields of each sample per experiment were taken to calculate the mean value. The mean values of three independent experiments of each group were used for statistical analysis.

\section{Live/dead assay}

Live/dead assay was performed to detect cell viability using the LIVE/DEAD ${ }^{\mathrm{ma}}$ Viability/Cytotoxicity Kit (Life Technologies GmbH, Darmstadt, Germany) according to the product description ${ }^{39,40}$. Images of six random fields of each sample per experiment were taken by the fluorescence microscope (Olympus) to calculate the mean value. The mean values of three independent experiments of each group were used for statistical analysis.

\section{Statistical analyses}

All experiments in our studies were repeated three times except for otherwise specified. Statistics were display with the mean \pm standard derivation. The data were statistically analyzed by the one-way analysis of variance (ANOVA) or Student's $t$-test, and the significant difference was set at $P \leq 0.05$.

\section{Results}

Knockdown of endogenous REST inhibits RPC proliferation but promotes differentiation

REST represses differentiation of ESCs, NPCs, bone marrow stromal cells, etc. ${ }^{11,41}$. However, the role of REST in the regulation of RPC fate is still unclear. To investigate the roles of REST in RPC proliferation and differentiation, we first detected the expression profile of REST in RPCs. As shown in Fig. 1a, REST protein was generally present in the nuclei of undifferentiated RPCs (under proliferation medium), and a significant reduction of REST expression was recorded after the siRNA of REST was designed and transfected into RPCs (Figs. 1a-d). Following Cell Counting Kit-8 (CCK-8) analysis, there was significantly inhibited expansion in siREST-treated cultures for the following $48 \mathrm{~h}$ and $72 \mathrm{~h}$, while there was no marked difference between the two groups on the first day (Fig. 1e), implying knockdown of the expression of REST reduce RPC proliferation. Further, the qPCR results showed that the expression levels of Ki-67 (a marker for cell proliferation ability) decreased notably (approximately 50\%) in siREST-treated RPC cultures compared with the control group (Fig. 1f). Then, the immunocytochemistry results showed that over $50 \%$ of the RPCs in the control group were positive for the cell proliferation markers BrdU and Ki-67 (Figs. 1g-i), which is indicative of active proliferation. The percentages of cells positive for BrdU and Ki-67 in the siREST group were measured to be approximately $42.25 \pm 1.35 \%$ and $41.57 \pm 2.77 \%$, respectively, which were significantly lower than those in the control group (Figs. 1g-i). However, compared with the control group, the percentage of nestin-positive cells exhibited no significant change although the immunoreactive intensity of nestin faded in siREST-treated RPC cultures (Fig. S1A-C). These results suggest that REST is important for sustaining RPC proliferation and interference of REST expression did not result in a loss of RPC multipotency.

Further study was focused on the effects of REST on RPC differentiation. Before detection of RPC differentiation markers, such as $\beta 3$-tubulin (a pan-neuronal marker), rhodopsin (the rod photoreceptor marker), recoverin (the 
A

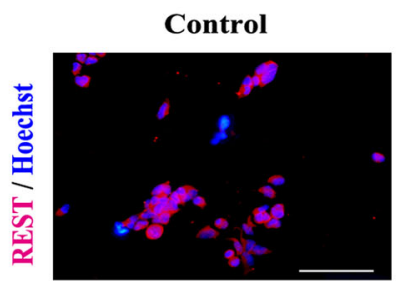

C

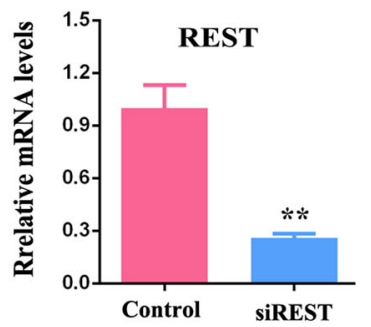

E

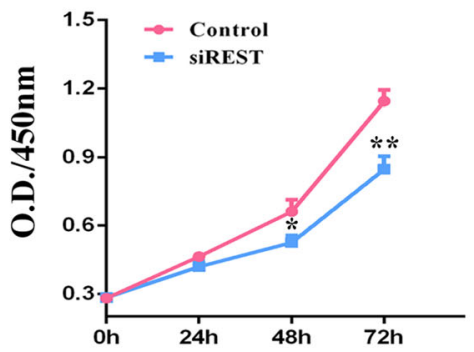

G

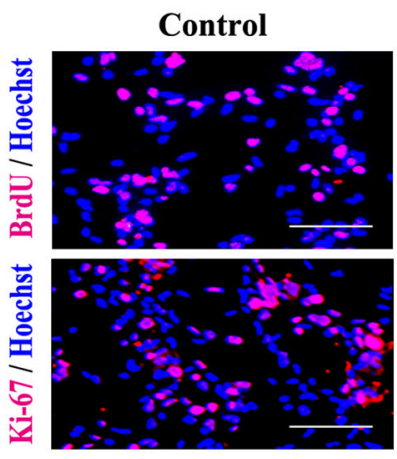

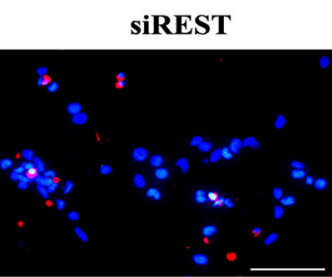

B

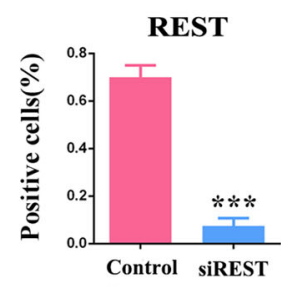

D

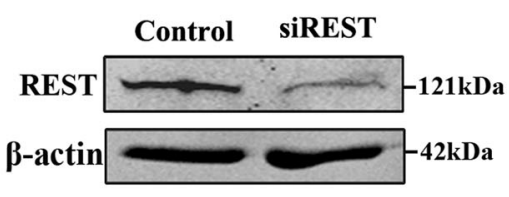

F

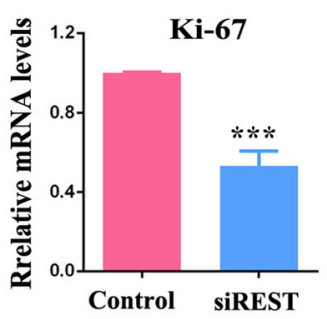

H

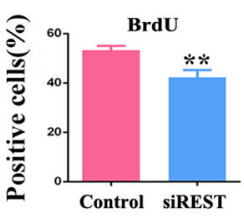

I

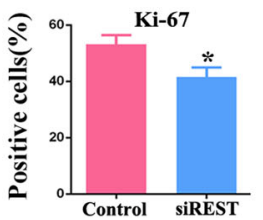

Fig. 1 siREST inhibits RPC proliferation. $\mathbf{a}$, $\mathbf{b}$ The immunocytochemistry, $\mathbf{c}$ qPCR analysis and $\mathbf{d}$ western blot results revealed that the expression levels of REST decreased in the siREST-treated RPC cultures compared with the control. e The proliferation ability of the RPCs was assessed via CCK-8 analysis. The proliferation ability of the RPCs markedly decreased after treatment with siREST under proliferation conditions. $\mathbf{f}$ According to the qPCR analysis, expression levels of Ki-67 decreased significantly in siREST-treated RPC cultures. $\mathbf{g}$-i Immunocytochemistry with antibodies against BrdU and Ki-67 revealed the effects of siREST on RPC proliferation and was consistent with the results shown above. Scale bars: $100 \mu \mathrm{m}$. Data are the averages of three independent experiments. Error bars indicate the standard error of the mean. ${ }^{*} P \leq 0.01,{ }^{* *} P \leq 0.01,{ }^{* *} P \leq 0.001$ (Student's $t$-test)

cone and rod photoreceptor marker), Brn3a (a marker of ganglion neurons) and calbindin (a marker for horizontal neurons), RPCs were cultured in differentiation medium without (control group) or with siREST (siREST group) treatment for 7 days. In the RPC cultures treated with siREST, the expression levels of $\beta 3$-tubulin, rhodopsin, recoverin, Brn3a and calbindin were obviously increased (Fig. 2a). The results of the western blot were consistent with our qPCR assay (Figs. 2b-e). In addition, immunocytochemistry analysis showed a marked increase in the $\beta 3$-tubulin and recoverin marker levels upon siREST treatment (Figs. 2f-h), indicating that knockdown of REST expression enhanced RPC neuronal differentiation. In the meanwhile, we detected the progenitor markers nestin and Pax-6. The prominent decrease of nestin and Pax- 6 expression implied that the process of differentiation was accelerated in siREST-treated cultures (Figs. 2i-k). Above all, these results suggest that REST plays a crucial role in RPC proliferation and differentiation. 

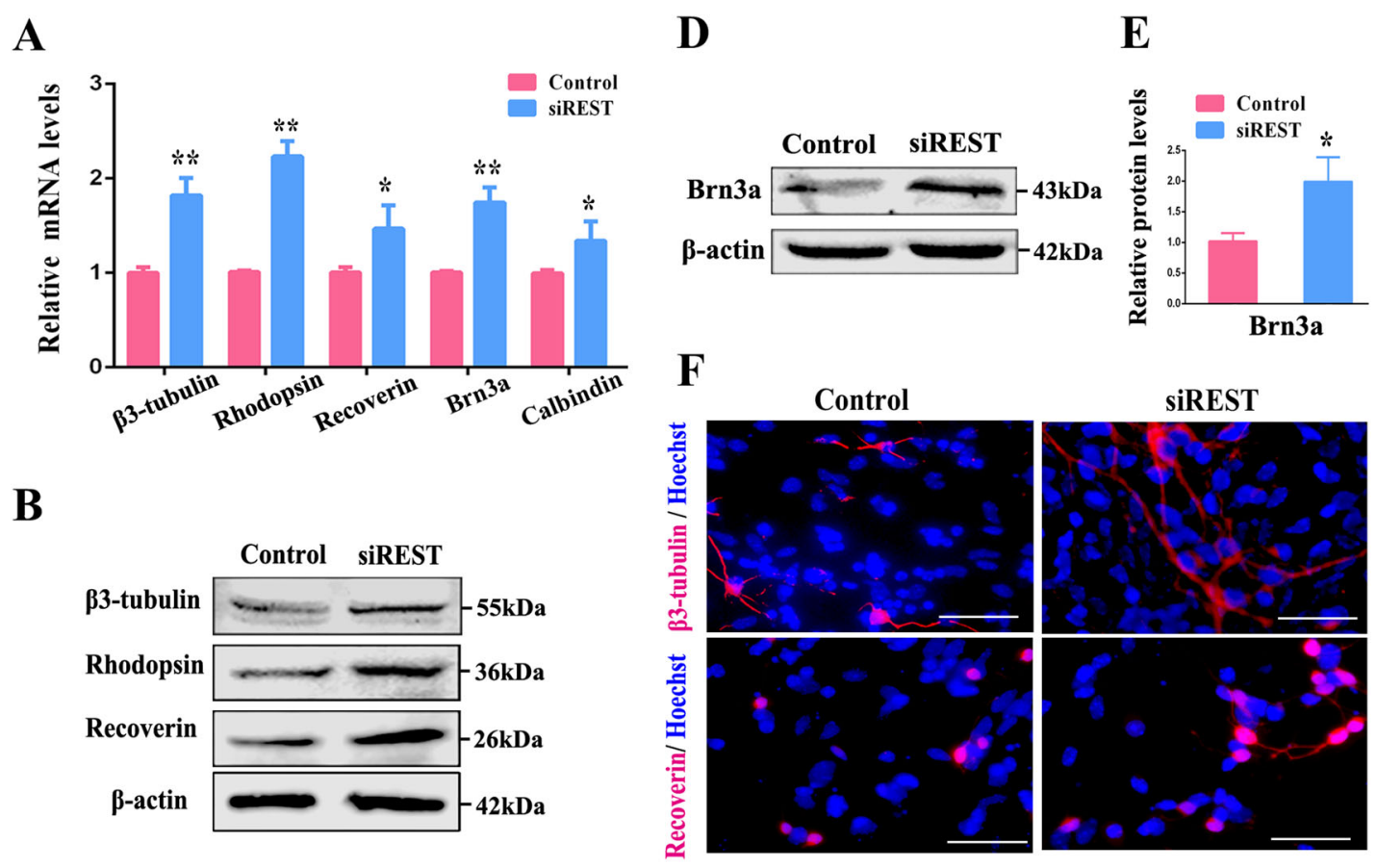

C

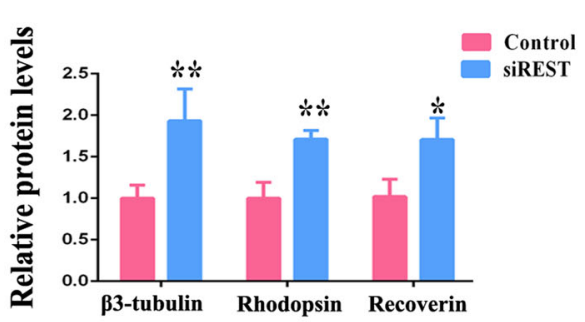

\section{G}

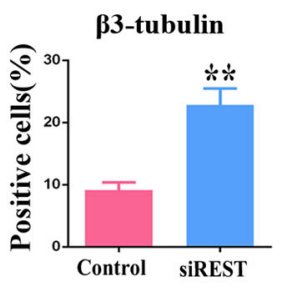

H

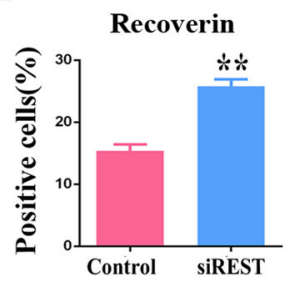

I

$\mathbf{J}$

\section{$\mathbf{K}$}
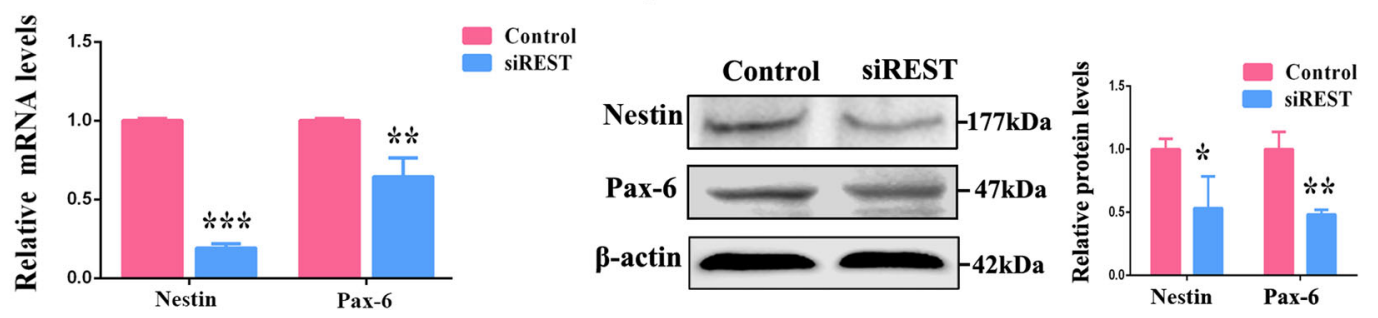

Fig. 2 siREST enhances RPC differentiation. a The expression levels of RPC differentiation-related markers, including $\beta 3$-tubulin (a pan-neuronal marker), rhodopsin (a marker for rod photoreceptors), recoverin (a marker for rod and cone photoreceptors), Brn3a (a marker for ganglion neurons) and calbindin (a marker for horizontal neurons), were elevated by siREST according to qPCR analysis. $\mathbf{b}$-e The results of western blot showed that the expression levels of $\beta 3$-tubulin, rhodopsin, recoverin and Brn3a were augmented in siREST-treated RPC cultures compared with the control. The western blot was scanned and normalized to $\beta$-actin. $\mathbf{f}$-h Immunocytochemistry showed that the percentages of $\beta 3$-tubulin- and recoverin-positive cells were markedly increased in the siREST-treated RPC cultures compared with the control. i qPCR results indicated that nestin and Pax-6 (retinal progenitor markers) were downregulated in siREST-treated RPC cultures. $\mathbf{j}-\mathbf{k}$ The expression levels of nestin and Pax- 6 were repressed after siREST treatment, according to the western blot. The western blot was scanned and normalized to $\beta$-actin. Scale bars: $50 \mu m$. Data are the averages of three independent experiments. Error bars indicate the standard error of the mean. ${ }^{*} P \leq 0.05$, ${ }^{* *} P \leq 0.01,{ }^{* * *} P \leq 0.001$ (Student's $t$-test)

\section{RA inhibits RPC proliferation and accelerates RPC differentiation}

REST is a repressor of neuronal genes whose expression is associated with poor neuronal differentiation ${ }^{42}$. As RA promotes neuronal differentiation, it may involve the modulation of REST expression. To detect the relationship between REST and RA in RPCs, the effects of RA on RPCs proliferation and differentiation were firstly determined. It is acknowledged that RA promoted photoreceptor differentiation in embryonic retinal and early 
A

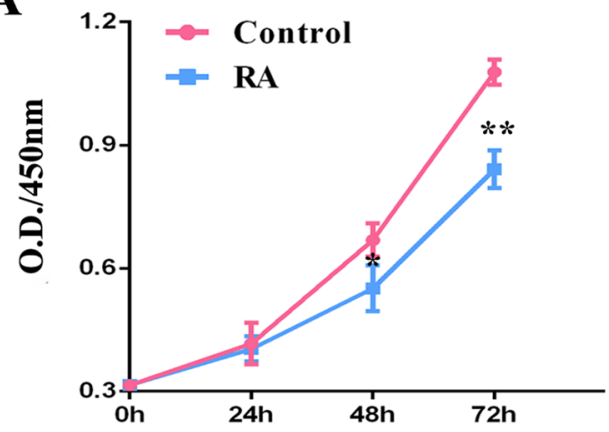

C
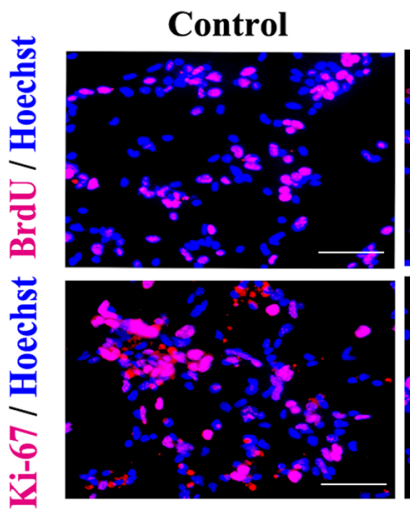
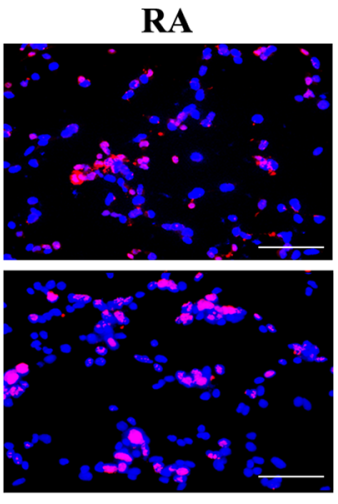

B

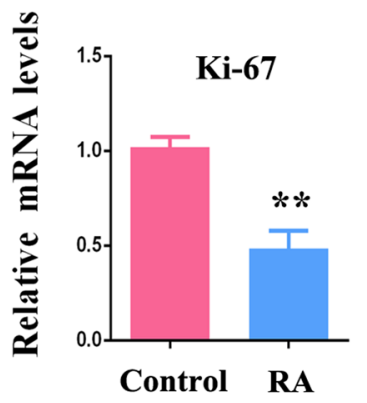

D

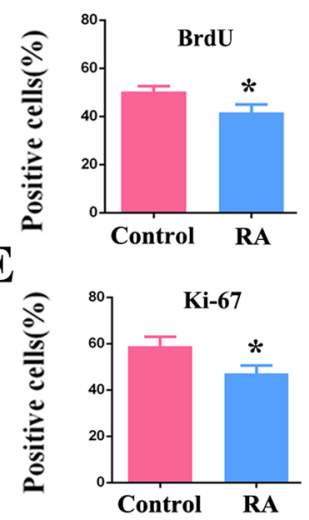

Fig. 3 RA inhibits RPC proliferation. a The proliferation ability of the RPC cultures was assessed via CCK-8 analysis. A weakened proliferation capacity was detected in the RA-treated RPCs when compared with control. bThe qPCR results revealed that the expression level of Ki-67 decreased in the RPC cultures treated with RA. c-e Immunochemistry showed that the percentages of BrdU- and Ki-67-positive cells in the RA-treated RPC cultures were significantly lower than those in the control group. Scale bars: $100 \mu \mathrm{m}$. Data are the averages of three independent experiments. Error bars indicate the standard deviation of the mean. ${ }^{*} P \leq 0.05,{ }^{*} P \leq 0.01$ (Student's $t$-test)

postnatal retinal cultures ${ }^{43}$. In this study, we detected the effects of RA on RPC proliferation and differentiation. To evaluate the effects of RA on RPC proliferation, RPCs were cultured with $100 \mathrm{ng} / \mathrm{ml}$ RA for 3 days under proliferation medium. The suppression on RPC expansion was detected by CCK- 8 and QPCR analysis, as shown in Figs. 3a, b. Additionally, as revealed in the immunocytochemistry analysis, the percentages of BrdU-positive and Ki-67-positive cells were significantly decreased in RA-treated RPC cultures (Figs. 3c-e). In addition, our data showed that the immunoreactive intensity of nestin in RA-treated RPC cultures is faded; however, the percentages of nestin-positive cells exhibited no significant difference between RA and control groups (Fig. S1D-F). Meanwhile, our data from qPCR and western blot showed that the significant difference of caspase-3 (a marker for cell apoptosis) expression level was not detected between RA and control group, which was consistent with our live/ dead assay (Fig. S2). These results implied that RA inhibited the proliferation ability of RPCs without inducing cell apoptosis and reducing their multipotency.
To determine the effect of RA on the differentiation of RPCs, the RPCs were treated with RA for 7 days under differentiation conditions. The expression levels of differentiation markers $\beta 3$-tubulin, rhodopsin, recoverin, Brn3a and calbindin were enhanced in RA-treated RPCs, according to the results of the qPCR and western blot (Figs. 4a-e). Additionally, the proportions of $\beta 3$-tubulinand recoverin-positive cells were increased in RA-treated RPC cultures when compared with the control group (Figs. 4f-h). Although nestin and Pax-6 showed an opposite trend, decreasing compared with the control group (Figs. 4i-k). Collectively, these data showed the effects of RA on RPC growth, which generally had the same tendencies as the knockdown of REST expression; that is, it inhibited proliferation but promoted differentiation, suggesting a negative correlation of REST and RA.

\section{RA downregulates the expression level of REST}

According to the above results, RA inhibited the proliferation but promoted the differentiation of RPCs, in 

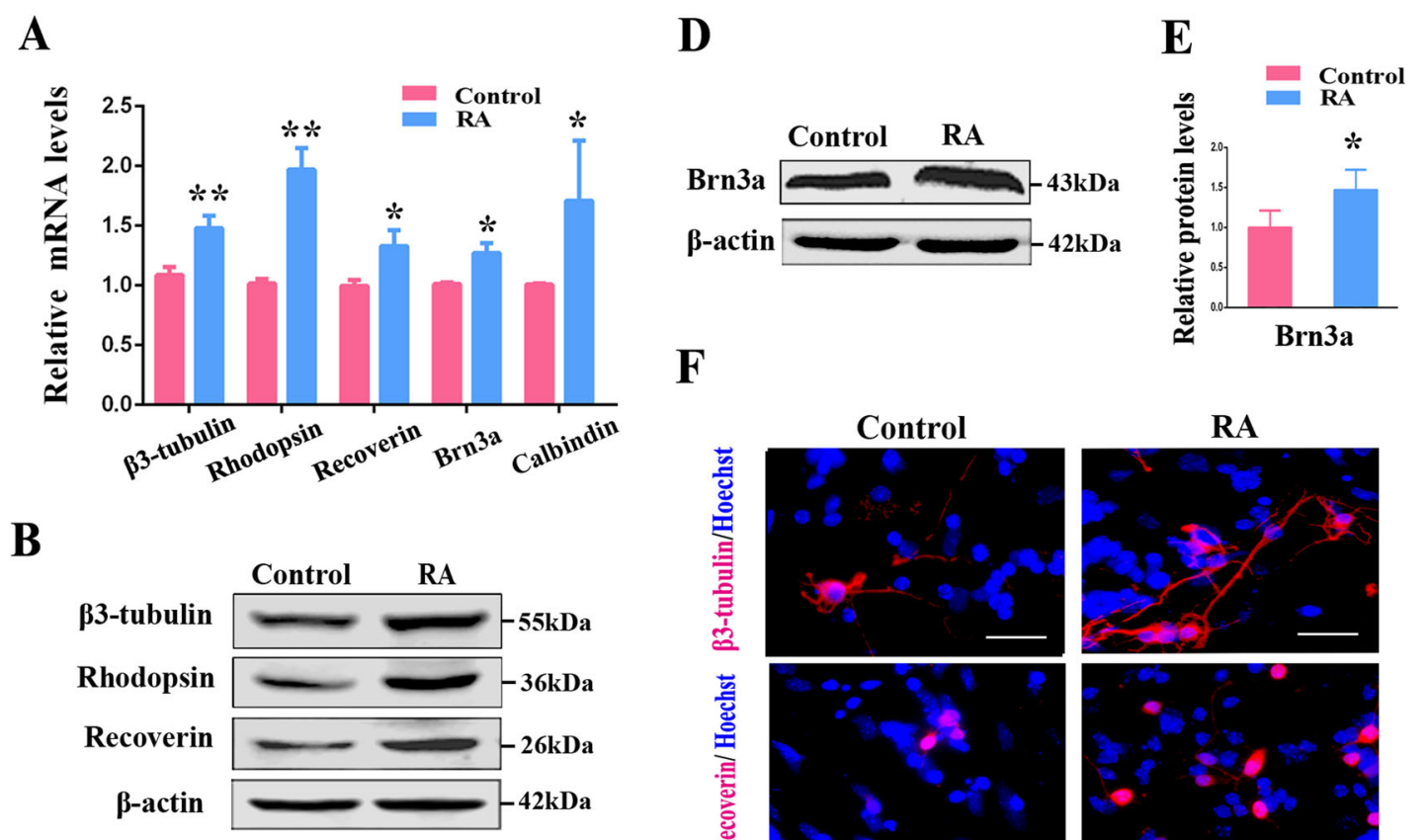

F

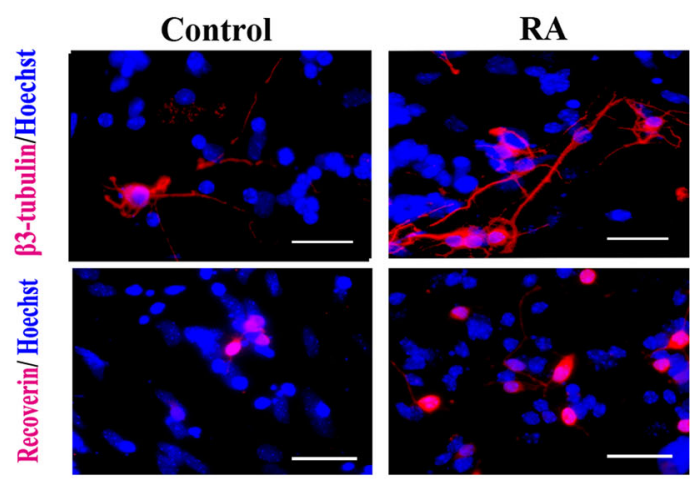

C

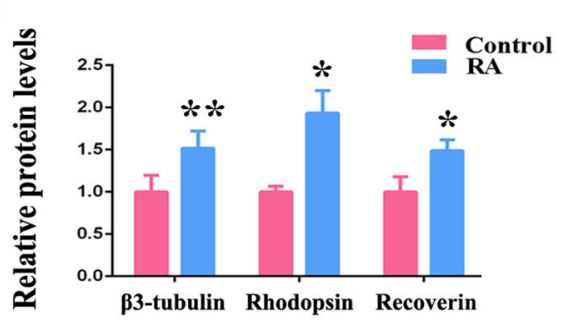

G

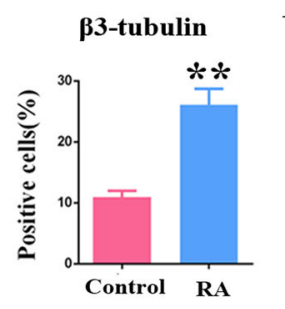

H
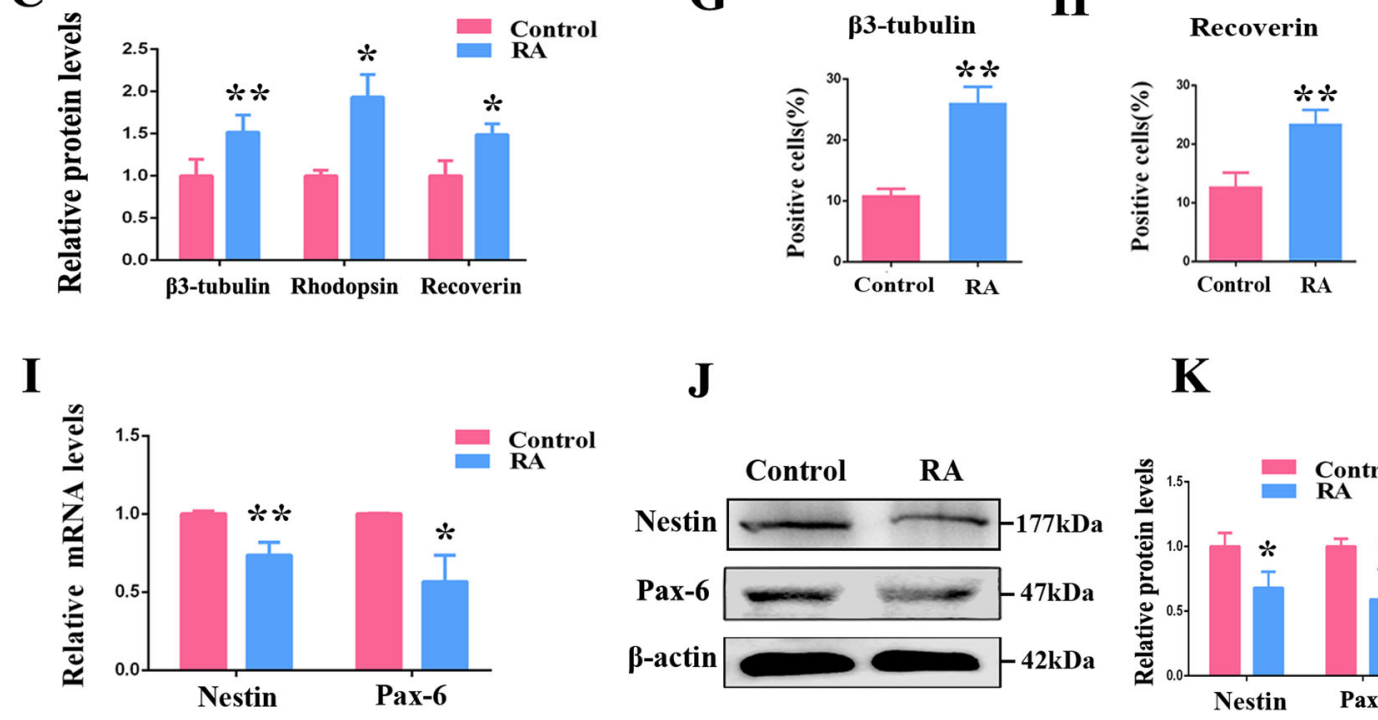

$\mathbf{J}$

K
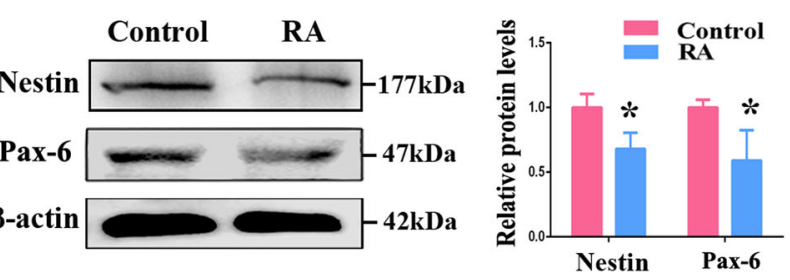

Fig. 4 RA enhances RPC differentiation. a The qPCR analysis showed that the expression levels of the RPC differentiation-related markers, including $\beta 3$-tubulin, rhodopsin, recoverin, Brn3a and calbindin, were elevated in RA-treated RPC cultures. b-e The results of the western blot revealed that $\beta 3$-tubulin, rhodopsin, recoverin and Brn3a were upregulated in RA-treated RPC cultures compared with the control. The western blot was scanned and normalized to $\beta$-actin. $\mathbf{f}$ - $\mathbf{h}$ Immunochemistry showed that the percentages of $\beta 3$-tubulin- and recoverin-positive cells in RA group were significantly higher than those in control group. $\mathbf{i}-\mathbf{k}$ According to the GPCR and western blot, the expression levels of nestin and Pax- 6 were repressed in the RA-treated RPC cultures. Scale bars: $50 \mu \mathrm{m}$. Data are the averages of three independent experiments. Error bars indicate the standard deviation of the mean. ${ }^{*} P \leq 0.05,{ }^{* *} P \leq 0.01$ (Student's $t$-test)

agreement with the siREST results. In the current study, we first detected the endogenous expression of REST during RPC differentiation. As the qPCR analysis disclosed, in comparison with day 0 , the expression levels of REST gradually decreased during RPC differentiation, dropping to approximately $40 \%$ at day 7 (Fig. 5a). Notably, the REST protein level also gradually decreased over time in the differentiation medium (Fig. 5b). To verify our speculation that RA is involved in the modulation of REST expression, RPCs were induced to differentiate with RA for 7 days. As the immunocytochemistry analysis showed, REST gradually decreased during differentiation, 


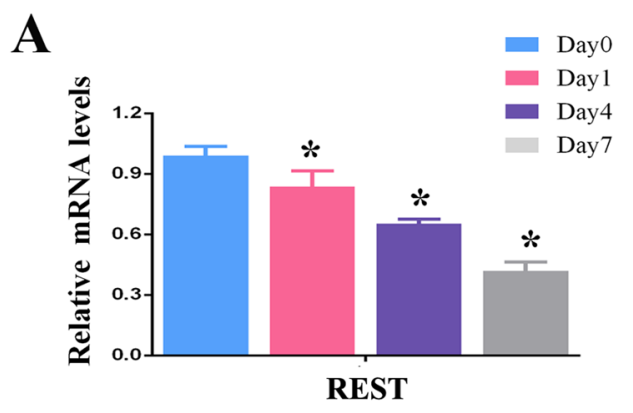

\section{B}

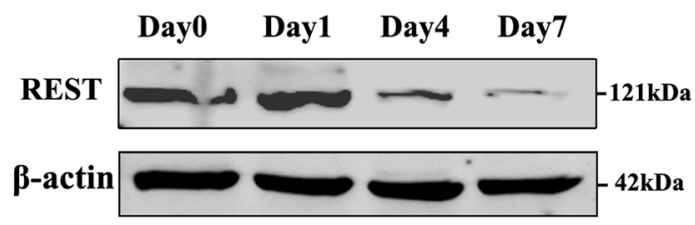

C

REST / Hoechst
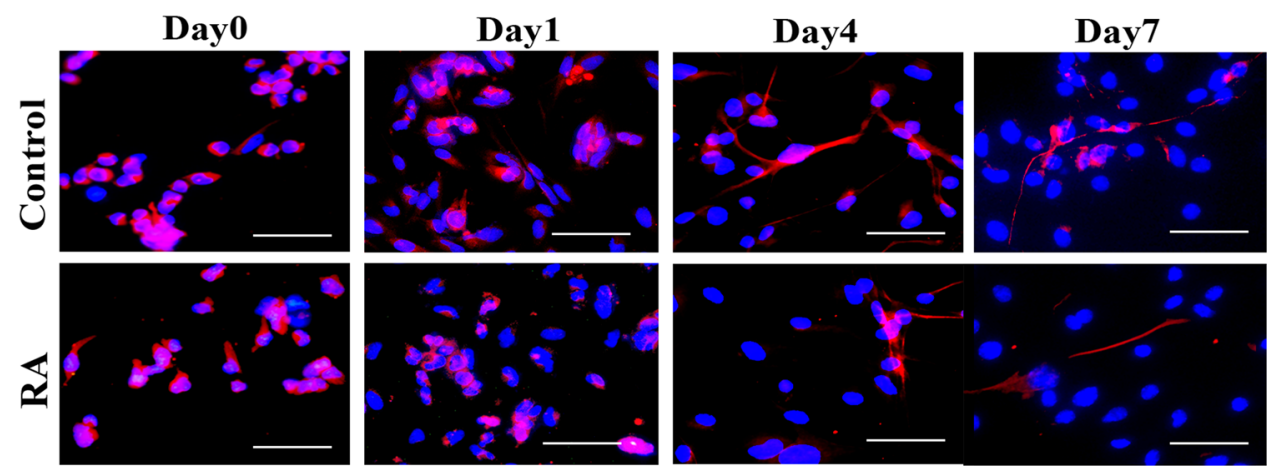

D

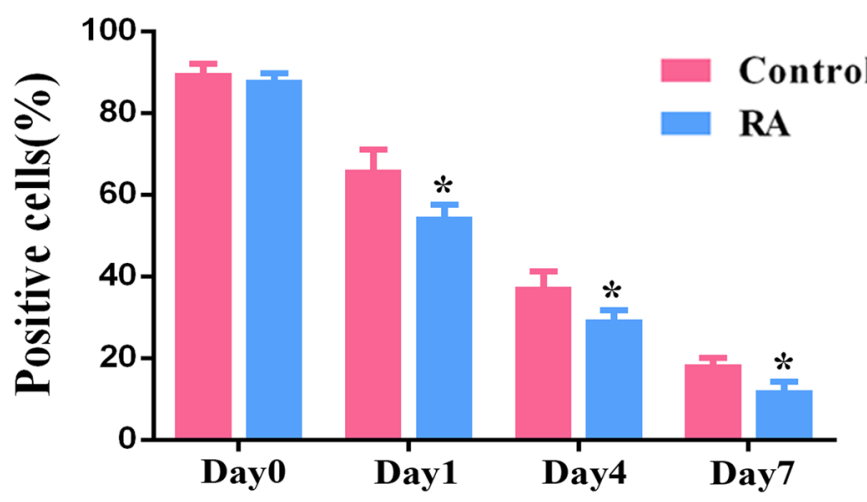

Fig. 5 RA downregulates REST protein expression. a The $\mathrm{QPCR}$ and $\mathbf{b}$ western blot results revealed that REST was gradually decreased during RPC differentiation. $\mathbf{c}$, $\mathbf{d}$ Immunocytochemistry showed that RA accelerated the decrease of REST protein levels during RPC differentiation. Data are the averages of three independent experiments. Scale bars: $50 \mu \mathrm{m}$. Error bars indicate the standard deviation of the mean. ${ }^{*} P \leq 0.05$ (Student's $t$-test and one-way ANOVA)

and the RA treatment could accelerate this reduction process (Figs. 5c, d). These results suggested that RA enhanced the downregulation of REST expression during RPC differentiation.

\section{REST regulated by RA through miR-29a and proteasomal degradation}

Based on the above results, we further explored how RA downregulated REST expression. A previous study disclosed that RA regulated neural gene expression through altering miRNA expression ${ }^{44}$. Meanwhile, REST was the target gene of miR-29a ${ }^{35}$. Thus, we speculated that, in RPCs, REST also interacted with miR-29a and that the regulation of REST by RA might involve miR-29a expression. The following experiment was first performed to detect whether miR-29a regulates the expression of REST in RPCs. In the current study, RPCs were transduced with the miR-29a mimics (pre-miR-29a group) or 

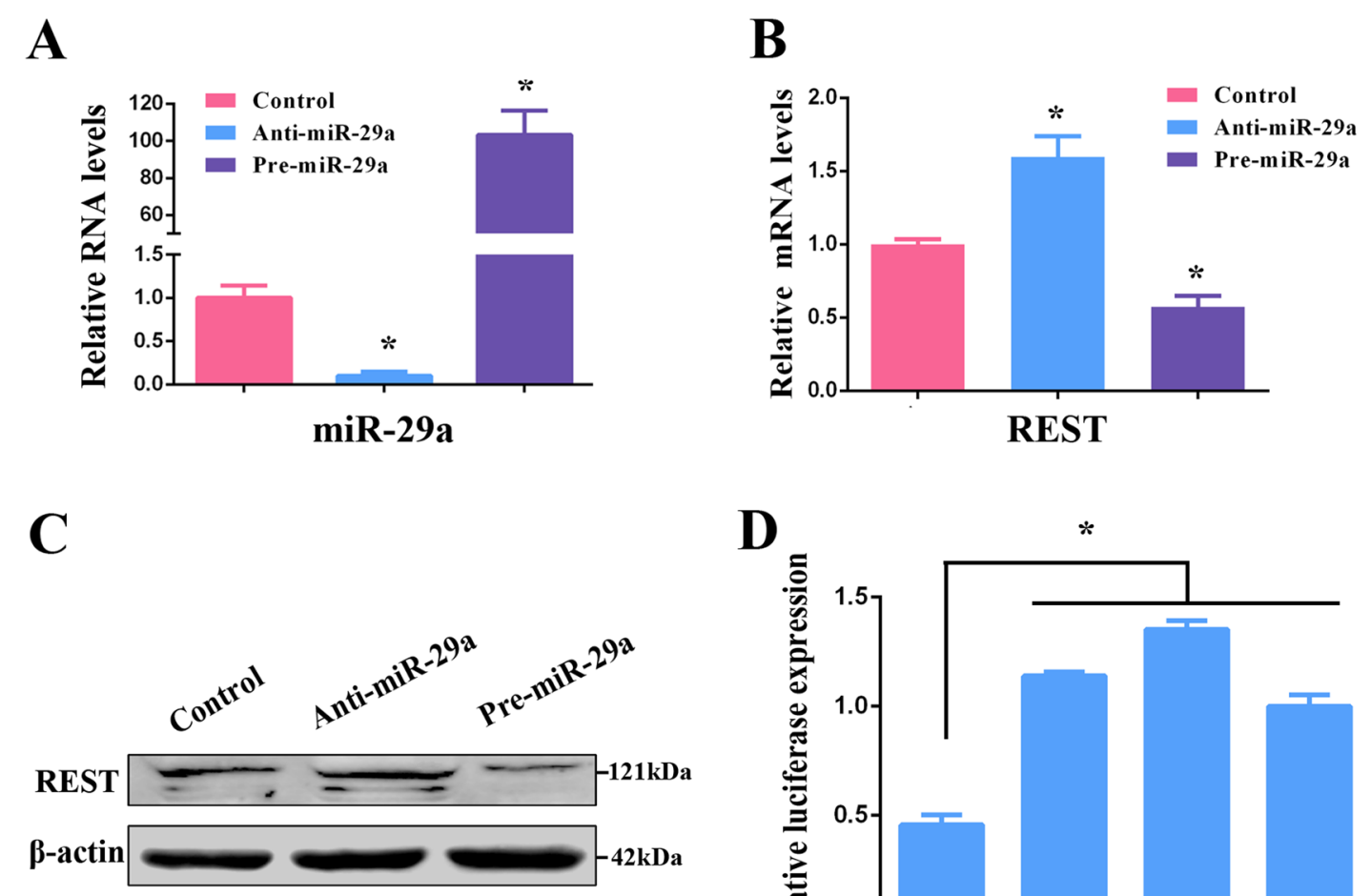

D

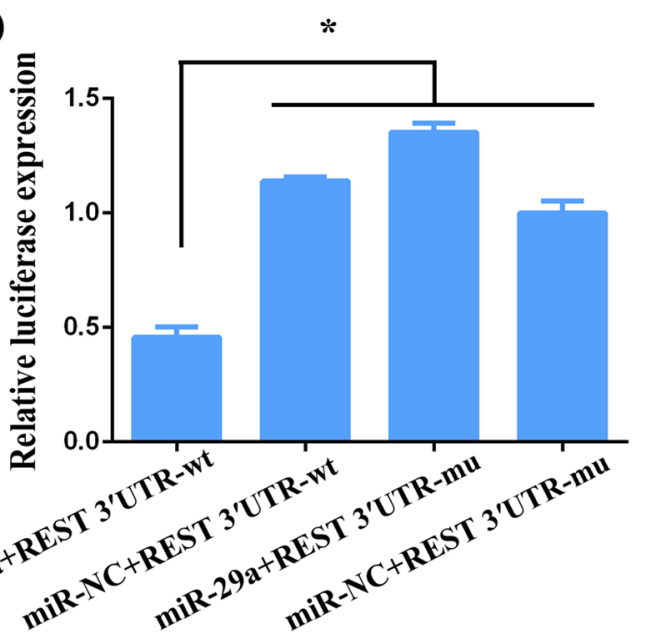

$\mathbf{E}$

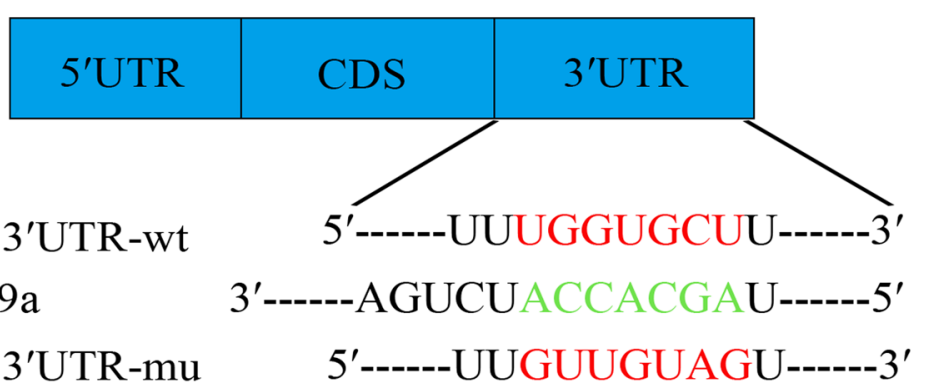

Fig. 6 REST is a target gene of miR-29a in RPCs. a The qPCR analysis showed that compared with control group, the expression levels of miR-29a were sharply downregulated by miR-29a inhibitor, and remarkably upregulated by miR-29a mimics. b, c According to the qPCR and western blot results, the REST expression level was downregulated by the overexpression of miR-29a but promoted by transfection with the miR-29a inhibitor. $\mathbf{d}$, $\mathbf{e}$ Positions 561-567 of the $3^{\prime}$-UTR of the REST mRNA (REST 3'-UTR-wt) or a mutated (REST $3^{\prime}$-UTR-mu) sequence was designed and inserted into the pGL3-control plasmids. The dual luciferase reporter system confirmed miR-29a binding to the wild-type 3'-UTR sequences of REST. Co-transfection of miR-29a and the REST wild-type $3^{\prime}$-UTR binding site (REST $3^{\prime}$-UTR-wt) dramatically reduced the luciferase activity compared with the other groups. The firefly luciferase activity data were normalized to Renilla luciferase activity as a control. Data are the averages of three independent experiments. Error bars indicate the standard deviation of the mean. ${ }^{*} P \leq 0.05$ (Student's $t$-test and one-way ANOVA)

inhibitor (anti-miR-29a group) in proliferation medium. The transfected efficiency of miR-29a was detected by qPCR (Fig. 6a). As shown in Fig. 6b, in the miR-29a mimic- and miR-29a inhibitor-transfected RPCs, REST was significantly transcriptionally suppressed and transcriptionally stimulated, respectively, suggesting that miR- 29a negatively regulated the expression of REST. The results of western blot also displayed that compared with the control group, the expression level of REST was downregulated by the miR-29a mimics but upregulated by the miR-29a inhibitor, respectively (Fig. 6c). These results indicate REST may be an active target gene of miR-29a in 
A

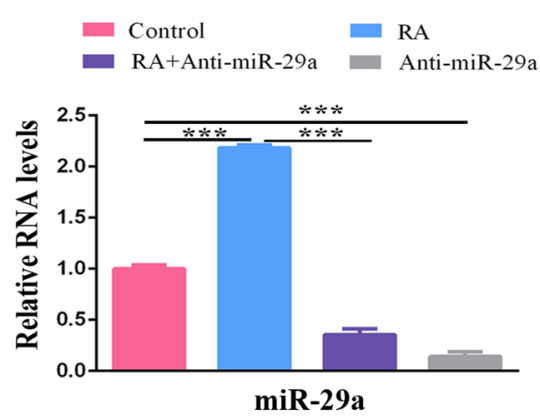

C

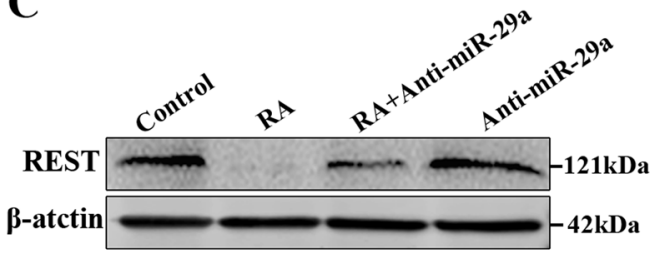

B

$$
\begin{array}{ll}
\text { Control } & \text { RA } \\
\text { RA+Anti-miR-29a } & \text { Anti-miR-29a }
\end{array}
$$

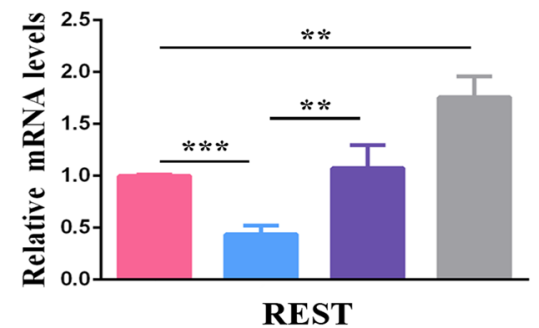

D

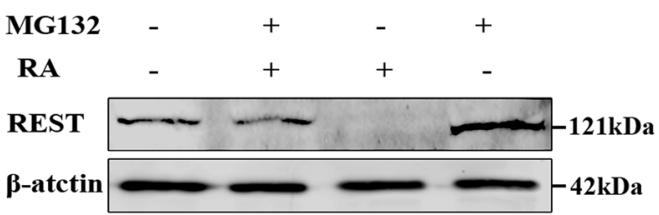

Fig. 7 RA inhibits REST expression through miR-29a and proteasome. a The expression level of miR-29a was remarkably elevated in RA-treated RPC cultures (compared with the control group), and this upregulation could be crippled by miR-29a inhibitor. b,c The qPCR analysis and western blot revealed that REST expression level was decreased in RA group (compared with the control group), and this downregulation could be rescued by miR-29a inhibitor. $\mathbf{d}$ The western blot showed the expression level of REST was reduced significantly in RA-treated RPC cultures (lane 3) compared with control (lane 1); this reduction could be reversed by MG-132 treatment (lane 2). Data are the averages of three independent experiments. Error bars indicate the standard deviation of the mean. ${ }^{* *} P \leq 0.01,{ }^{* *} P \leq 0.001$ (Student's $t$-test)

RPC growth. To further verify that miR-29a binds to the REST mRNA 3'-UTR to regulate the translational progress, a luciferase reporter was performed. REST $3^{\prime}$-UTR containing the miR-29a binding site (REST $3^{\prime}$-UTR-wt) and its mutant (REST $3^{\prime}$-UTR-mu) at the positions 561-567 were cloned downstream of the firefly luciferase coding sequence in the pGL3-control vector. The luciferase assay showed that the luciferase activity was dramatically decreased during co-transfection of miR-29a and REST 3'-UTR-wt compared with the other groups (Fig. 6d), indicating that positions $571-576$ of the REST 3'-UTR were a direct target of miR-29a (Fig. 6e). These data indicate that miR-29a regulates REST expression by directly binding to the REST 3'-UTR.

We next tested whether RA can regulate the expression of miR-29a and further alter the REST expression level. RPCs were cultured under proliferation medium without (control group) or with RA (RA group), RA plus miR-29a inhibitor (RA + miR-29a inhibitor group) or miR-29a inhibitor (miR-29a inhibitor group) for 3 days. It was worth noting that, as we expected, according to the qPCR analysis, miR-29a was upregulated remarkably in the RPC cultures treated only with RA compared with the control group, and this can be reversed by adding the miR-29a inhibitor (Fig. 7a), indicating that RA promotes the expression of miR-29a and that this promotion could be blocked by the miR-29a inhibitor. Conversely, the expression of REST was downregulated to $50 \%$ in RAtreated RPCs compared with the control, and this suppression could be reversed by the miR-29a inhibitor (Fig. 7b). The western blot analysis was consistent with the qPCR results (Fig. 7c). The above data suggest that REST could be mediated by RA through upregulation of miR-29a.

Importantly, the expression of REST in the culture treated with both RA and miR-29a inhibitor (Fig. 7c-lane 3) was significantly more than that in the RPC cultures treated only with RA (Fig. 7c-lane 2) but still less than that in the control (Fig. 7c-lane 1), indicating that other mechanisms might be involved in this process. In general, there are two main pathways to downregulate REST protein expression, decrease its generation (transcriptional level) and accelerate its degradation (posttranscriptional level). We then treated RPCs with the proteasomal inhibitor MG-132 $(20 \mu \mathrm{M})$ for $8 \mathrm{~h}$ in the presence and absence of RA (under proliferation conditions). The protein level of REST was significantly rescued by MG-132 (in RPC cultures treated with both RA and MG-132, Fig. 7d-lane 2) compared the cells treated with RA alone (Fig. 7d-lane 3), demonstrating that blocking the proteasomal activity could counter the RA-mediated degradation of the REST protein.

To further determine whether the effect of RA on RPC proliferation and differentiation was through miR-29a, CCK- 8 and qPCR analyses were performed. In proliferation medium, the RA-induced RPC proliferation inhibition could be partially rescued by the miR-29a inhibitor, according to the CCK-8 analysis (Fig. 8a). The qPCR 

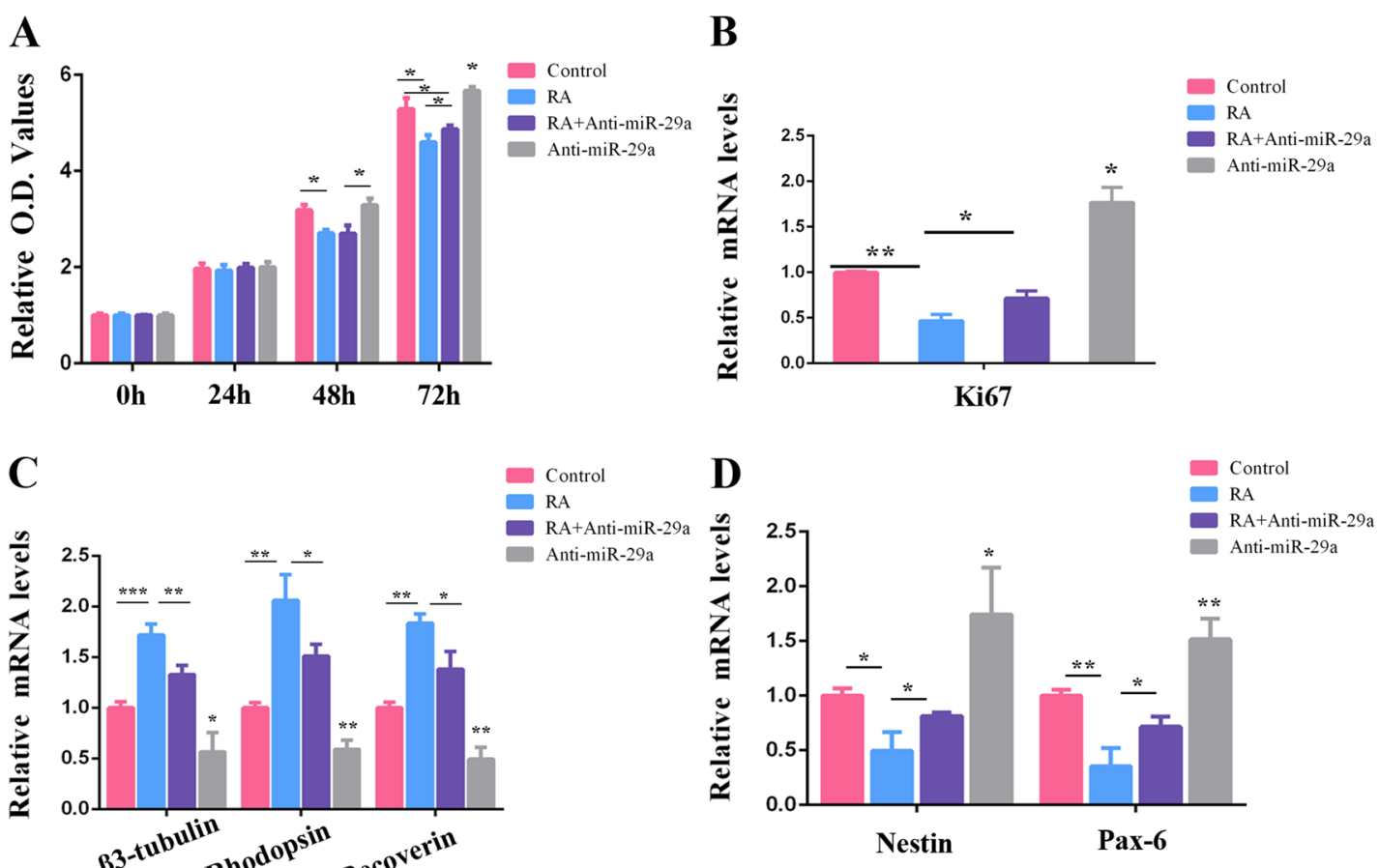

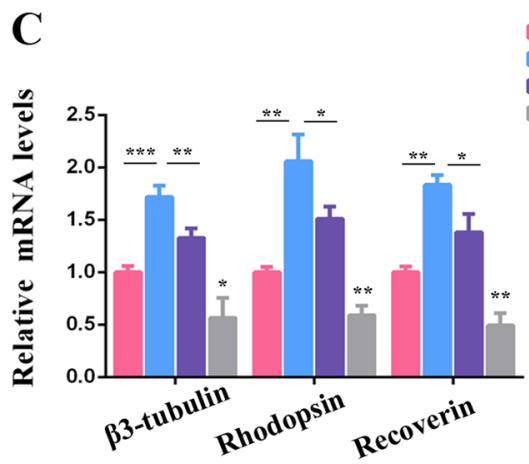

$\mathbf{E}$

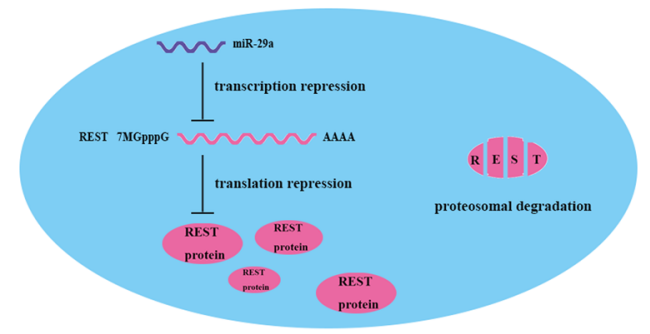

Control

RA

RA+Anti-miR-29a
Normal RPC

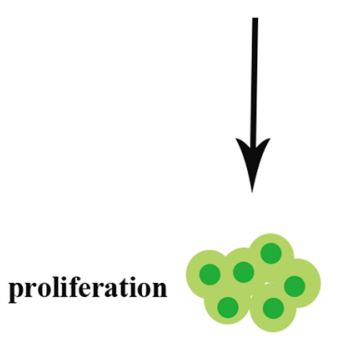

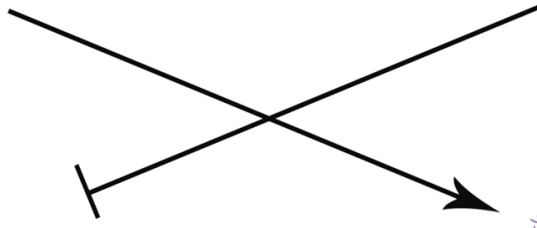

differentiation
RA treated RPC

RA
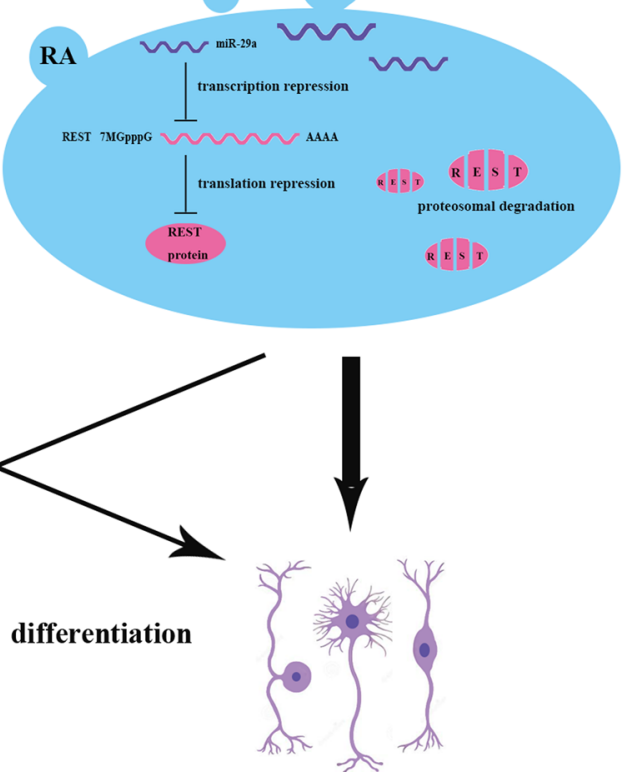

Fig. 8 RA regulates RPC proliferation and differentiation through miR-29a. a The results of the CCK-8 analysis showed that RA induced RPC proliferation inhibition, and this trend could be partially rescued by the miR-29a inhibitor. $\mathbf{b}$ The GPCR analysis showed that the expression of Ki-67 was upregulated in RPCs treated with both RA and the miR-29a inhibitor compared with those only treated with RA. c The expression levels of RPC differentiation markers (B3-tubulin, rhodopsin and recoverin) were increased RA-treated RPC cultures (compared with control cultures), and miR-29a inhibitor partially reversed RA induced RPC differentiation markers upregulation. $\mathbf{d}$ Compared with RPCs only treated with RA, the expression levels of nestin and Pax- 6 were upregulated in RPCs treated with both RA and the miR-29a inhibitor, according to the qPCR analysis. e A model of the role of REST mediated by RA in the regulation of RPC proliferation and differentiation. RA could directly degrade REST protein through the proteasome and indirectly suppress REST gene expression through the upregulation of miR-29a. Data are the averages of three independent experiments. Error bars indicate the standard deviation of the mean. ${ }^{*} P \leq 0.05,{ }^{* *} P \leq 0.01,{ }^{* * *} P \leq 0.001$ (Student's $t$-test) 
analysis showed that the expression level of the cell proliferation marker Ki-67 was suppressed by approximately $50 \%$ in the RA group compared with the control group, and this suppression was rescued partially by the miR-29a inhibitor (in RA + miR-29a inhibitor group, Fig. 8b). Additionally, RA-induced neuronal differentiation promotion (under differentiation medium) was also crippled by adding miR-29a inhibitor (Fig. 8c). Furthermore, compared with RPCs only treated with RA, the expression levels of nestin and Pax-6 were also upregulated in RPCs treated with both RA and the miR-29a inhibitor, according to the qPCR analysis (Fig. 8d). Therefore, we concluded that RA inhibited the proliferation and induced the differentiation of RPCs partly through promoting miR29a expression.

Taken together, our data suggest that REST can enhance proliferation of RPCs but restricts their differentiation abilities and that the expression of REST can be mediated by RA at both the transcriptional and posttranscriptional levels (Fig. 8e).

\section{Discussion}

RPCs are a subset of multipotent cells that are characterized by capacities of self-renewal and differentiation into retinal neurons. As a promising resource for cellbased therapy in the treatment of retinal degenerative diseases, such as AMD and RP, the primary obstacles of RPCs currently are their limited proliferation and differentiation ability into specific retinal neurons in vitro ${ }^{45,46}$. In this study, our results indicate that REST is a conserved, neuron-suppressed protein that could be modulated by RA through miR-29a and proteasomal degradation, respectively, to regulate the proliferation and differentiation of RPCs.

REST has been recognized as a repressor of neuronal genes during neurogenesis ${ }^{47}$. It is known to be expressed in the nuclei of different types of cells and bind RE-1 to restrain terminal neuronal differentiation-related genes ${ }^{47-49}$. Thus, REST was present at its highest level in the nuclei of pluripotent ESCs and was gradually downregulated and translocated into the cytoplasm with the maturation of neurons ${ }^{13,50}$. In this study, REST was also found to be highly expressed in nuclei in proliferation medium and translocated into the cytoplasm when it was downregulated during RPC differentiation. In ESCs and NPCs, REST played a role in maintaining their multipotency and the direct knockdown of REST to promote differentiation $^{11,42}$. Our study demonstrated the self-renewal abilities of RPCs, which were also impaired after knocking down REST expression, whereas the marker expression for retinal neurons was upregulated. In addition, the percentages of nestin-positive cells in siREST-treated RPC cultures exhibited no significant change, although the immunoreactive intensity of nestin faded (Fig. S1), implying that interference of REST expression did not result in a loss of RPC multipotency, which is consistent with a previous study ${ }^{42}$. These results indicated that REST in RPCs, as in ESCs and NPCs, plays an important role in maintaining cell proliferation and restricting their differentiation.

As direct knockdown of endogenous REST reduced the RPC proliferation but accelerated the RPC differentiation, which phenocopied the observed effects of RA on RPCs, we speculated that RA is involved in REST regulation. Our results showed that the REST protein levels could be significantly downregulated by RA, and two mechanisms were involved in this process, including decrease in the production and promotion of the degradation of REST protein. In ESCs, previous study reported that RA regulated mRNA transcription by suppressing the expression levels of miR-200b and miR-200c $c^{44}$. In this study, we showed, in RPCs, that RA could inhibit REST mRNA expression partly through enhancing the expression of miR-29a (the luciferase report verified that REST is a target gene of miR-29a, consistent with a previous study $^{35}$ ). Our further study showed that RA-induced RPC proliferation inhibition and differentiation promotion could be crippled by the miR-29a inhibitor, which, in turn, negatively mediated REST expression, implying that RA worked on RPCs through miR-29a. It is known that a miRNA usually has multiple target genes, Zhang et al. reported that miR-29a could promote RPC differentiation and attenuated RPC proliferation by negatively regulating the expression of RBM8a (one target gene of miR-29a) ${ }^{37}$, indicating that RBM8a may have a role in RA regulating RPC proliferation and differentiation. In addition, REST was also reported to be regulated by RA through a posttranscriptional mechanism mediated by proteasomal degradation $^{51}$, and RA can upregulate the expression level of REST-specific E3-ligase (Skp1-Cul1-F-box protein complex containing the F-box protein $\beta$-TRCP, SCF $\beta$ TRCP), which promote REST proteasomal degradation in neuroblastoma cells ${ }^{12,51}$. In the current study, REST protein levels were substantially increased in the presence of the proteasome inhibitor MG-132 plus RA group compared with the levels in the cells treated with RA alone. The reversal of RA-mediated REST degradation by MG-132 treatment indicates the proteasomal involvement in modulating the REST levels in RPCs. However, other mechanisms may be also involved in RA regulating RPC proliferation and differentiation. Previous studies displayed that RA promoted neural differentiation at the expense of proliferation through upregulated the proneural and neurogenic transcription factors ${ }^{52,53}$, but downregulated pro-proliferation transcription factors ${ }^{54-57}$. RA may also work on its receptors (RARs) to regulate the direct downstream target genes, which are involved in the cell proliferation-differentiation switch $^{53,58,59}$. Taken 
together, our data demonstrated that RA induced REST suppression through miR-29a-modulated transcriptional and proteasome-modulated posttranscriptional levels. RA, as a recognized secure and effective clinical medicine in the treatment of various diseases, is supposed to be applied in future retinal degeneration therapy.

This study discloses a novel functional protein, REST, which governs RPC proliferation and differentiation, can be mediated by RA, which may be instructive for the application of RPC transplantation in the treatment of retinal degeneration diseases. Further studies will focus on elucidating the roles of REST and RA in retinal development and their application in vivo to treat retinal degeneration diseases.

\section{Conclusion}

In this study, our data demonstrated that REST plays an important role in RPC proliferation and differentiation. Knockdown of REST expression inhibits proliferation of RPCs but stimulates their differentiation into specific retinal neurons, implying that REST can be proposed as a novel biomarker for evaluating RPC behavior. Another key finding is that REST expression could be regulated at the transcriptional and posttranscriptional levels by RA through elevating miR-29a expression and accelerating proteasomal degradation, respectively. The current study provides us with new sights in controlling RPC fates, although the effects of REST in vivo remain to be explored.

\section{Acknowledgements}

The research was supported by Shanghai Municipal Education CommissionGaofeng Clinical Medicine Grant Support (20161316), the National Natural Science Foundations of China (81570883 and 31500835) and The Science and Technology Commission of Shanghai (17DZ2260100).

\section{Author details}

'Department of Ophthalmology, Ninth People's Hospital, Shanghai Jiao Tong University School of Medicine, 200011 Shanghai, P.R. China. ${ }^{2}$ Shanghai Key Laboratory of Orbital Diseases and Ocular Oncology, 200011 Shanghai, P.R. China

\section{Conflict of interest}

The authors declare that they have no conflict of interest.

\section{Publisher's note}

Springer Nature remains neutral with regard to jurisdictional claims in published maps and institutional affiliations.

Supplementary Information accompanies this paper at https://doi.org/ 10.1038/s41419-018-0473-5.

Received: 2 December 2017 Revised: 7 March 2018 Accepted: 12 March 2018

Published online: 18 April 2018

\footnotetext{
References

1. Klassen, H., Sakaguchi, D. S. \& Young, M. J. Stem cells and retinal repair. Prog. Retin. Eye Res. 23, 149-181 (2004).
}

2. Klassen, $\mathrm{H}$. J. et al. Multipotent retinal progenitors express developmental markers, differentiate into retinal neurons, and preserve light-mediated behavior. Invest. Ophthalmol. Vis. Sci. 45, 4167-4173 (2004).

3. Yang, P., Seiler, M. J., Aramant, R. B. \& Whittemore, S. R. Differential lineage restriction of rat retinal progenitor cells in vitro and in vivo. J. Neurosci. Res. 69 , 466-476 (2002)

4. Xia, J. et al. An in vitro comparison of two different subpopulations of retinal progenitor cells for self-renewal and multipotentiality. Brain Res. 1433, 38-46 (2012).

5. Yang, P., Seiler, M. J., Aramant, R. B. \& Whittemore, S. R. In vitro isolation and expansion of human retinal progenitor cells. Exp. Neurol. 177, 326-331 (2002).

6. Gu, P. et al. Isolation of retinal progenitor and stem cells from the porcine eye. Mol. Vis. 13, 1045-1057 (2007)

7. Mortazavi, A., Leeper Thompson, E. C., Garcia, S. T., Myers, R. M. \& Wold, B. Comparative genomics modeling of the NRSF/REST repressor network: from single conserved sites to genome-wide repertoire. Genome Res. 16, 1208-1221 (2006)

8. Otto, S. J. et al. A new binding motif for the transcriptional repressor REST uncovers large gene networks devoted to neuronal functions. J. Neurosci. 27, 6729-6739 (2007)

9. Johnson, D. S., Mortazavi, A., Myers, R. M. \& Wold, B. Genome-wide mapping of in vivo protein-DNA interactions. Science 316, 1497-1502 (2007).

10. Ballas, N. et al. Regulation of neuronal traits by a novel transcriptional complex. Neuron 31, 353-365 (2001).

11. Ballas, N., Grunseich, C., Lu, D. D., Speh, J. C. \& Mandel, G. REST and its corepressors mediate plasticity of neuronal gene chromatin throughout neurogenesis. Cell 121, 645-657 (2005).

12. Westbrook, T. F. et al. SCFbeta-TRCP controls oncogenic transformation and neural differentiation through REST degradation. Nature 452, 370-374 (2008).

13. Singh, S. K., Kagalwala, M. N., Parker-Thornburg, J., Adams, H. \& Majumder, S. REST maintains self-renewal and pluripotency of embryonic stem cells. Nature 453, 223-227 (2008).

14. Paquette, A. J., Perez, S. E. \& Anderson, D. J. Constitutive expression of the neuron-restrictive silencer factor (NRSF)/REST in differentiating neurons disrupts neuronal gene expression and causes axon pathfinding errors in vivo. Proc. Natl. Acad. Sci. USA 97, 12318-12323 (2000).

15. Lu, T. et al. REST and stress resistance in ageing and Alzheimer's disease. Nature 507, 448-454 (2014)

16. McClelland, S. et al. Neuron-restrictive silencer factor-mediated hyperpolarization- activated cyclic nucleotide gated channelopathy in experimental temporal lobe epilepsy. Ann. Neurol. 70, 454-464 (2011).

17. Calderone, A. et al. Ischemic insults derepress the gene silencer REST in neurons destined to die. J. Neurosci. 23, 2112-2121 (2003).

18. Formisano, L. et al. Ischemic insults promote epigenetic reprogramming of mu opioid receptor expression in hippocampal neurons. Proc. Natl. Acad. Sci. USA 104, 4170-4175 (2007).

19. Tateno, M., Ukai, W., Hashimoto, E., Ikeda, H. \& Saito, T. Implication of increased NRSF/REST binding activity in the mechanism of ethanol inhibition of neuronal differentiation. J. Neural Transm. Nienna) 113, 283-293 (2006).

20. Napoli, J. L. Physiological insights into all-trans-retinoic acid biosynthesis. Biochim. Biophys. Acta 1821, 152-167 (2012).

21. Maden, M. Retinoic acid in the development, regeneration and maintenance of the nervous system. Nat. Rev. Neurosci. 8, 755-765 (2007).

22. Bastien, J. \& Rochette-Egly, C. Nuclear retinoid receptors and the transcription of retinoid-target genes. Gene 328, 1-16 (2004).

23. Christie, V. B. et al. Retinoid supplementation of differentiating human neural progenitors and embryonic stem cells leads to enhanced neurogenesis in vitro. J. Neurosci. Methods 193, 239-245 (2010).

24. Grondona, J. M. et al. Retinal dysplasia and degeneration in RARbeta2/ RARgamma2 compound mutant mice. Development 122, 2173-2188 (1996).

25. Kelley, M. W., Turner, J. K. \& Reh, T. A. Retinoic acid promotes differentiation of photoreceptors in vitro. Development 120, 2091-2102 (1994).

26. Kelley, M. W., Williams, R. C., Turner, J. K, Creech-Kraft, J. M. \& Reh, T. A. Retinoic acid promotes rod photoreceptor differentiation in rat retina in vivo. Neuroreport 10, 2389-2394 (1999).

27. Osakada, F. et al. Toward the generation of rod and cone photoreceptors from mouse, monkey and human embryonic stem cells. Nat. Biotechnol. 26, 215-224 (2008)

28. Chapman, M. S. Vitamin a: history, current uses, and controversies. Semin Cutan. Med Surg. 31, 11-16 (2012). 
29. Herget, T., Specht, H., Esdar, C., Oehrlein, S. A. \& Maelicke, A. Retinoic acid induces apoptosis-associated neural differentiation of a murine teratocarcinoma cell line. J. Neurochem. 70, 47-58 (1998).

30. Hecht, A. et al. Validation of a molecular risk score for prognosis of patients with acute promyelocytic leukemia treated with all-trans retinoic acid and chemotherapy-containing regimens. Clin. Lymphoma Myeloma Leuk. 17, 889-896 (2017).

31. Patrick, E., Buckley, M., Muller, S., Lin, D. M. \& Yang, J. Y. Inferring data-specific micro-RNA function through the joint ranking of micro-RNA and pathways from matched micro-RNA and gene expression data. Bioinformatics $\mathbf{3 1}$ 2822-2828 (2015).

32. Kriegel, A. J., Liu, Y., Fang, Y., Ding, X. \& Liang, M. The miR-29 family: genomics, cell biology, and relevance to renal and cardiovascular injury. Physiol. Genom. 44, 237-244 (2012).

33. Wang, $H$. et al. NF-kappaB-YY1-miR-29 regulatory circuitry in skeletal myogenesis and rhabdomyosarcoma. Cancer Cell 14, 369-381 (2008).

34. Li, Z. et al. Biological functions of miR-29b contribute to positive regulation of osteoblast differentiation. J. Biol. Chem. 284, 15676-15684 (2009).

35. Duan, P. et al. miR-29a modulates neuronal differentiation through targeting REST in mesenchymal stem cells. PLOS ONE 9, e97684 (2014).

36. Quintero, H., Gomez-Montalvo, A. I. \& Lamas, M. MicroRNA changes through Muller glia dedifferentiation and early/late rod photoreceptor differentiation. Neuroscience 316, 109-121 (2016).

37. Zhang, Y. et al. miR-29a regulates the proliferation and differentiation of retinal progenitors by targeting Rbm8a. Oncotarget 8, 31993-32008 (2017).

38. Zhang, D. et al. Betacellulin regulates the proliferation and differentiation of retinal progenitor cells in vitro. J. Cell Mol. Med 22, 330-345 (2017).

39. Zhou, S., Cui, Z. \& Urban, J. Dead cell counts during serum cultivation are underestimated by the fluorescent live/dead assay. Biotechnol. J. 6, 513-518 (2011).

40. Jung, Y., Klein, O. J., Wang, H. \& Evans, C. L. Longitudinal, label-free, quantitative tracking of cell death and viability in a 3D tumor model with OCT. Sci. Rep. 6, 27017 (2016).

41. Lunyak, V. V. \& Rosenfeld, M. G. No rest for REST: REST/NRSF regulation of neurogenesis. Cell 121, 499-501 (2005).

42. Thakore-Shah, K., Koleilat, T., Jan, M., John, A. \& Pyle, A. D. REST/NRSF knockdown alters survival, lineage differentiation and signaling in human embryonic stem cells. PLoS ONE 10, e0145280 (2015).

43. Watanabe, T. \& Raff, M. C. Diffusible rod-promoting signals in the developing rat retina. Development 114, 899-906 (1992).
44. Zhang, J. et al. Retinoic acid induces embryonic stem cell differentiation by altering both encoding RNA and microRNA expression. PLOS ONE 10, e0132566 (2015).

45. Klassen, $\mathrm{H}$. Stem cells in clinical trials for treatment of retinal degeneration. Expert Opin. Biol. Ther. 16, 7-14 (2016).

46. Luo, J. et al. Human retinal progenitor cell transplantation preserves vision. J. Biol. Chem. 289, 6362-6371 (2014).

47. Chong, J. A. et al. REST: a mammalian silencer protein that restricts sodium channel gene expression to neurons. Cell 80, 949-957 (1995).

48. Gopalakrishnan, V. REST and the RESTless: in stem cells and beyond. Future Neurol. 4, 317-329 (2009).

49. Majumder, S. REST in good times and bad: roles in tumor suppressor and oncogenic activities. Cell Cycle 5, 1929-1935 (2006).

50. Buckley, N. J., Johnson, R., Sun, Y. M. \& Stanton, L. W. Is REST a regulator of pluripotency? Nature 457, E5-E6 (2009).

51. Singh, A. et al. Retinoic acid induces REST degradation and neuronal differentiation by modulating the expression of SCF(beta-TRCP) in neuroblastoma cells. Cancer 117, 5189-5202 (2011).

52. Rogers, C. D., Moody, S. A. \& Casey, E. S. Neural induction and factors that stabilize a neural fate. Birth Defects Res C. Embryo Today 87, 249-262 (2009).

53. Moody, S. A., Klein, S. L., Karpinski, B. A., Maynard, T. M. \& Lamantia, A. S. On becoming neural: what the embryo can tell us about differentiating neural stem cells. Am. J. Stem Cells 2, 74-94 (2013).

54. Franco, P. G., Paganelli, A. R., Lopez, S. L. \& Carrasco, A. E. Functional association of retinoic acid and hedgehog signaling in Xenopus primary neurogenesis. Development 126, 4257-4265 (1999).

55. Janesick, A. et al. ERF and ETV3L are retinoic acid-inducible repressors required for primary neurogenesis. Development 140, 3095-3106 (2013).

56. Diez del Corral, R. et al. Opposing FGF and retinoid pathways control ventral neural pattern, neuronal differentiation, and segmentation during body axis extension. Neuron 40, 65-79 (2003).

57. Wills, A. E., Choi, V. M., Bennett, M. J., Khokha, M. K. \& Harland, R. M. BMP antagonists and FGF signaling contribute to different domains of the neural plate in Xenopus. Dev. Biol. 337, 335-350 (2010).

58. Matsuo, T. \& Thiele, C. J. p27Kip1: a key mediator of retinoic acid induced growth arrest in the SMS-KCNR human neuroblastoma cell line. Oncogene 16, 3337-3343 (1998).

59. Sasaki, K. et al. Expression and role ofp27(kip1) in neuronal differentiation of embryonal carcinoma cells. Brain Res. Mol. Brain Res. 77, 209-221 (2000). 